\title{
Clinical Relevance of Serum Nortriptyline and 10-Hydroxy-Nortriptyline Measurements in the Depressed Elderly: A Multicenter Pharmacokinetic and Pharmacodynamic Study
}

\author{
N. M. K. Ng Ying Kin, Ph.D., N. Klitgaard, Ph.D., N. P. V. Nair, M.D., M. Amin, M.D., \\ P. Kragh-Sorensen, M.D., G. Schwartz, M.Sc., S. K. Ahmed, M.D., P. Holm, M.Sc., \\ C. Katona, M.D., and K. Stage, M.D.
}

In a recent placebo-controlled multicenter study, 38 patients, ranging in age between 62 and 88 years (median, 71) were treated with nortriptyline (NT) for up to 7 weeks. NT was administered in a dizided dose of $75 \mathrm{mg}$ daily and serum NT (se NT), and its 10-hydroxy-metabolites (se $\mathrm{OH}$ NT) were determined at various intervals. Several clinical measures of efficacy, including the 17-item Hamilton Rating Scale for Depression, were evaluated weekly as well as side effects (anticholinergic) and electrocardiogram (ECG) changes. Eighty-one percent of patients had NT levels in the previously defined therapeutic range of 50 to $170 \mathrm{ng} / \mathrm{mll}$, with steady state reached between 1 and 3 weeks. There was little individual variation in drug kinetics

KEY WORDS: Nortriptyline; 10-Hydroxynortriptyline; Blood levels; Efficacy; Anticholinergic side effects; Geriatric depression

The neuropsychopharmacology of the tricyclic antidepressant nortriptyline (NT) has been extensively studied in the nonelderly patient population but less so in the

From Douglas Hospital Research Centre and Department of Psychiatry (NNYK; NPVN; MA; GS; SKA), McGill University, Montréal, Québec, Canada; Odense University Hospital (NK; PK-S; KS), Odense, Denmark; Roche a/s (PH), Copenhagen, Denmark; Princess Alexandra Hospital (CK), London, England.

Address correspondence to N. M. K. Ng Ying Kin, Ph.D., Douglas Hospital Research Centre, 6875 LaSalle Boulevard, Verdun, Canada $\mathrm{H} 4 \mathrm{H} 1 \mathrm{R} 3$.

Received June 20, 1995; accepted July 19, 1995 and metabolism over the study period. In general se OH-NT levels were not greater than those of se NT.

Pharmacodynamic analyses showed that patients with moderate to severe anticholinergic side effects [CSE $(+)]$ had significantly higher NT levels than those with mild or no symptoms [CSE $(-)]$. Furthermore, repeated-measures ANOVA modeled over time showed a highly significant decrease in clinical measures in both CSE groups of patients and also a highly significant group-time interaction. Higher se OH-NT levels were associated with less anticholinergic side effects. No ECG changes were observed. [Neuropsychopharmacology 15:1-6, 1996]

elderly (Rubin et al. 1985; Georgotas et al. 1986, 1987a, 1987b; Katz et al. 1989; Miller et al. 1991). A therapeutic window for serum (se NT) in the range of 50 to $150 \mathrm{ng} / \mathrm{ml}$ has been proposed (Asberg et al. 1971; Kragh-Sorensen et al. 1973), and the peripheral anticholinergic side effects of this drug are well documented (Rafaelson et al. 1981; Potter et al. 1991). However, the relationship between serum concentrations of NT, its metabolite OH-NT, and anticholinergic side effects remains to be established, although a relationship between serum drug concentrations and ECG abnormalities has been reported by Young et al. (1985). In addition, the general pharmacokinetics of NT in the elderly depressed needs to be further elaborated. As part of a recent placebo-controlled double-blind multicenter study (Nair et al. 1995), el- 
derly depressed patients were treated with NT or placebo for up to 7 weeks. We report on the clinical relevance of serum NT and OH-NT measurements obtained during that trial.

\section{MATERIALS AND METHODS}

\section{Patients}

Seventy-three patients who fulfilled the DSM-III-R criteria for major depression (APA 1987) were treated with either NT ( $n=38$; age range, $62-68$; median, 71 years) or placebo $(n=35$; age range, $62-68$; median, 71 years) in a double-blind randomized fashion at three investigational sites in Canada, Denmark, and England as part of larger study (Nair et al. 1995).

Inclusion criteria at baseline involved a minimum total score of 18 on the first 17 items of the Hamilton Rating Scale for Depression (HRS-D; Hamilton, 1960). The duration of the current episode had to have lasted at least 4 weeks, with at least "moderately severe" rating on the Clinician's Global Assessment of Severity Scale (CGAS). The patients had received no tricyclic antidepressants during the preceding week, no monoamine oxidase inhibitors (MAOIs), and no neuroleptics during the previous 2 weeks, and no sleep deprivation or electroconvulsive therapy during the previous month. They also had no history of drug or alcohol abuse, known severe systemic diseases, acute infections, or clinically significant abnormal laboratory findings, including $a b$ normal ECG and blood pressure readings.

\section{Treatment Schedule}

The initial dose of NT was $25 \mathrm{mg}$ on the first evening, increased to $25 \mathrm{mg}$ in the morning and $50 \mathrm{mg}$ in the evening from the third day onward. Depending on the se NT level at the end of week 1 , the dose could be adjusted at the end of week 2 according to a procedure that kept the clinicians and the patients blind. If se NT levels were between 50 and $170 \mathrm{ng} / \mathrm{ml}$, the dose remained unchanged. If se NT levels went below $50 \mathrm{ng} /$ $\mathrm{ml}$, the morning dose was increased to $50 \mathrm{mg}$, with the evening dose unchanged. If se NT levels were above $170 \mathrm{ng} / \mathrm{ml}$, the evening dose was reduced to $25 \mathrm{mg}$. Thereafter, these adjusted doses remained constant throughout the study.

\section{Serum NT and Serum OH-NT Determinations}

Blood was collected in an additive-free Vacutainer tube at 4:00 p.m. before the afternoon dose at baseline, at the end of weeks 1 and 3 and at the end of treatment (up to end of week 7). Se NT and se OH-NT were assayed using a slightly modified version of a high-performance liquid chromatographic (HPLC) method described by Klitgaard (1977). Blood samples for the determinations of se-OH-NT were not available for all the subjects en- rolled in the study. Blood specimens from England and Denmark were assayed at Odense University Hospital, and those from Canada, at the Douglas Hospital Research Centre. Prior to the start of the study, an interintralaboratory validation was carried out on the results obtained from the assays of quality control human serum samples containing three different NT concentrations $(50,100$, and $170 \mathrm{ng} / \mathrm{ml})$. Four samples of each concentration were analyzed on three different days. The overall intralaboratory/interday reproducibility was 5.0 and 4.9 for Canada and Denmark, respectively. Two-way analysis of variance (ANOVA) with replicates and cross-classification according to country and concentrations confirm the linearity in the range of therapeutic concentrations analyzed for both laboratories; no differences between laboratory means were detected $(F=0.09, p>.8)$. The lower level of detection was 10 $\mathrm{ng} / \mathrm{ml}$, and the reproducibility of the assay was $5 \%$ at the $50-\mathrm{ng} / \mathrm{ml}$ level to $4 \%$ at the $170-\mathrm{ng} / \mathrm{ml}$ level.

\section{Clinical Assessments}

At baseline, at weekly intervals and finally at termination of the study, all patients were rated on the HRS-D, HMES (HRS-D with melancholia; Bech 1981), Clinical Global Assessments of Severity (CGAS), of Efficacy (CGAE), and of Tolerance (CGAT) scales and assessed for the incidence, duration, and severity (rated on a 3-point scale: mild, moderate, or severe) of anticholinergic events such as tremor, dry mouth, dry throat, blurred vision, constipation, sweating, micturition disturbances, and confusion. A checklist of adverse events was also used. ECGs were recorded at baseline and at treatment termination.

\section{Statistical Analyses}

All data were analyzed by ANOVA and repeated-measures ANOVA model over time, using the SPSS/PC+ program (version 5.0). Data on clinical measures used the last observation carried forward method. Except for the pharmacokinetic analysis, the data were subjected to a standard analysis of efficacy for those who stayed in the study for at least 3 weeks.

\section{RESULTS}

\section{Pharmacokinetics}

Of the 32 patients who completed the first week of treatment, only 6 had serum NT levels outside the previously defined therapeutic window ( 3 with levels below $50 \mathrm{ng} / \mathrm{ml}$ and 3 above $170 \mathrm{ng} / \mathrm{ml}$ ). Their dosages were adjusted accordingly at week 2 of treatment, as indicated. These patients, as well as two who were noncompliant and/or dropped out before week 3, were not included in the pharmacokinetic analysis.

Figure 1 shows the serum levels of NT and OH-NT at 
weeks 1 and 3 and at termination (days 29-45). Serum NT levels for 22 patients treated beyond week 3 were significantly higher at the end of week 3 than at the end of week 1 (means $\pm \mathrm{SD}(\mathrm{ng} / \mathrm{ml}), 118 \pm 53$ versus $100 \pm$ 33; $p<.02$ ). However, no significant differences between the metabolite levels at these two time points were found. The serum NT and OH-NT levels at termination were not significantly different from those at week 3 . On the other hand, there were significant correlations between the serum NT levels at weeks 1 and 3 and also between those at week 3 and termination. This was also the case for OH-NT. The following results were obtained: NT, weeks 1 and $3, r=0.81, p<.0001$; weeks 3 and termination, $r=.83, p<.001$. OH-NT: weeks 1 and $3, r=0.88, p<.0001$; weeks 3 and termination, $r=0.66, p<.03$.

On average serum OH-NT levels were equal to or less than serum NT levels at all three time points. There was a significant positive correlation between the serum levels of the parent drug and its metabolites at week $1(r=.49, p<.05)$. However, although positive correlations were also found at week 3 and termination, respectively $(r=0.30$ and 0.39$)$, these were not statistically significant.

\section{Pharmacodynamics}

Clinical Efficacy. Remission, defined as HRS-D total score below 10 at the end of treatment, was $41 \%$ (12/ $29)$, compared to 10 percent (3/31) for those on placebo.
The $95 \%$ confidence interval limits for the observed $+32 \%$ difference between nortriptyline and placebo were $+11 \%$ and $+52 \%$. For completers (observed cases analysis), the remission rate was $60 \%$ for nortriptyline compared to $11 \%$ for placebo.

Relationship between Clinical Efficacy Scores and Serum Levels of NT and its Metabolites. No significant associations were found between any of the following clinical efficacy measures: HRS-D, HMES, CGAE, or CGAS, and the serum NT, serum OH-T, or serum NT + OH-NT levels at week 3 and at termination, although serum levels of the metabolite, but not of the parent drug, were significantly associated with better tolerance as measured on the CGAT scale $(r=0.52, p<.02)$.

Adverse Events. The overall incidence of adverse events observed was $92 \%$ for patients on nortriptyline compared to $83 \%$ on placebo. However, for anticholinergic side effects they were 74 and 49 percent, respectively, and for orthostatic events, 42 and 26 percent, respectively. The intensity of the anticholinergic side effects was more severe with the active drug (median score of 2.1 on a scale of 1 to 3) than with the placebo (median score, 1.6). For orthostatic events, the respective numbers were 1.8 and 1.4 .

Side effects associated with cardiovascular functions (hypertension, edema in legs, and palpitation) were mild and infrequent, and there were no drug-induced abnormalities in electrocardiograms.

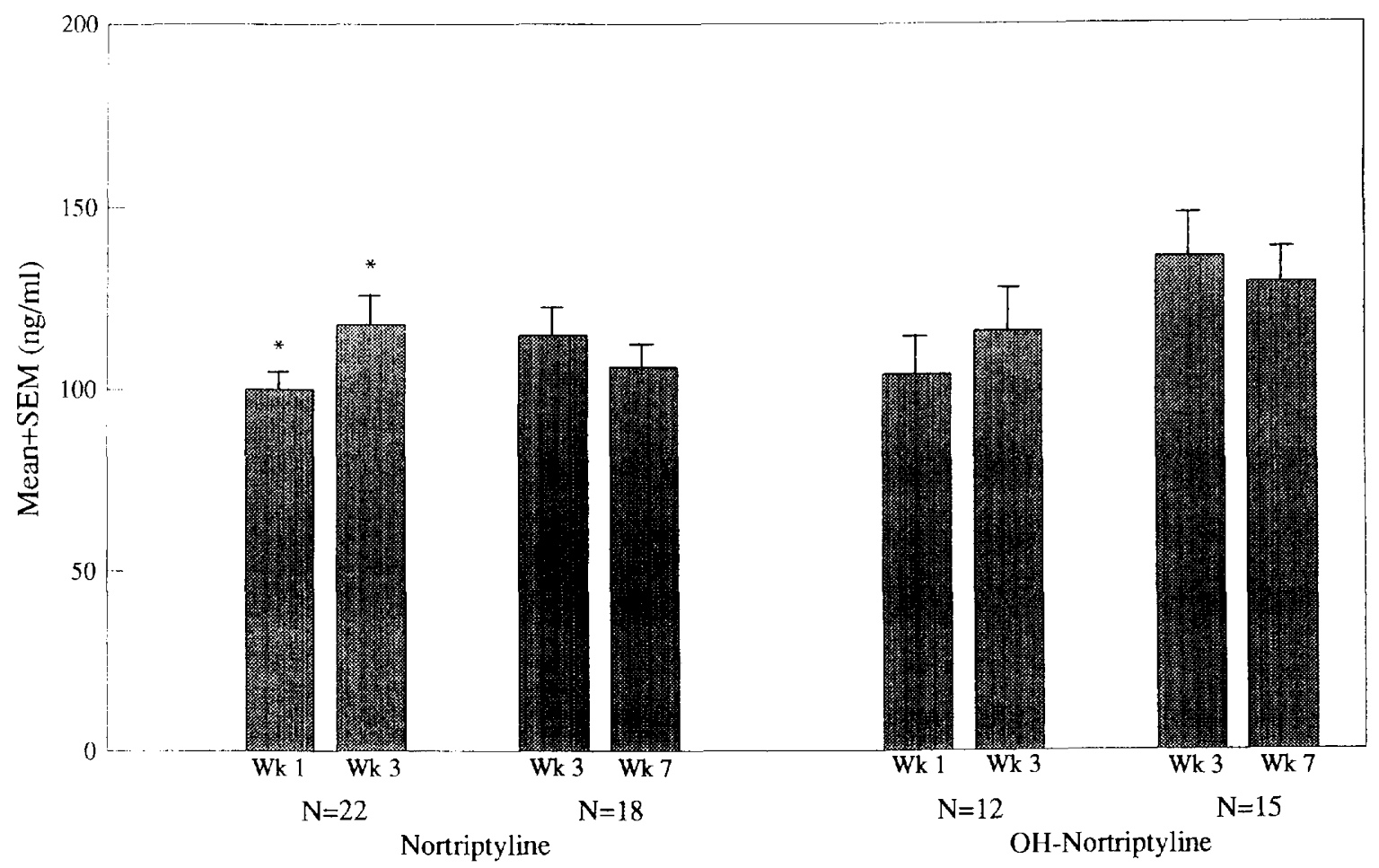

Figure 1. Serum levels of NT and OH-NT $\left(\mathrm{ng} / \mathrm{ml}\right.$ ) at weeks 1 and 3 and at termination (week 7 ). ${ }^{*} p<.02$. 
Relationship between NT Levels and Anticholinergic Side Effects. Table 1 shows the relationship between serum NT and serum OH-NT levels and the incidence and severity of anticholinergic side effects. For an analysis of the relationship between anticholinergic side effects and serum NT levels on the one hand, and clinical efficacy measures on the other, patients were divided into two groups: $\operatorname{CSE}(+)$, moderate to severe anticholinergic symptoms (score $>1$ ), and $\operatorname{CSE}(-)$, mild or absence of anticholinergic symptoms. The mean \pm SD serum NT levels of the $\operatorname{CSE}(+)$ group were significantly higher than those of the $\operatorname{CSE}(-)$ group at both week 1 $(n=32)$ and week $3(n=29)$, respectively $124 \pm 74$ vs. $82 \pm 35, p<.05$, and $133 \pm 64$ vs. $93 \pm 32, p<.05$.

Relationship between Metabolite (OH-NT) Levels and Anticholinergic Side Effects. In contrast to findings for the NT levels, there was no significant difference in the serum OH-NT levels between the CSE $(+)$ and $(-)$ groups at either time point. On the other hand, the ratios of serum level of metabolite to parent drug was significantly lower in the $\operatorname{CSE}(+)$ group at week $3(n=22)$; $0.83 \pm 0.28$ vs. $1.5 \pm 0.87 ;(p<.02)$. At week 1 , this ratio was also lower with a trend to statistical significance $(p<.07)$.

Relationship between Clinical Efficacy Scores and Anticholinergic Side Effects. One- and two-way repeated-measures ANOVAs of the clinical rating scales of efficacy used in this study were carried out over the 7-week period of the study for the $\operatorname{CSE}(+)$ and $\operatorname{CSE}(-)$ groups. In the $\operatorname{CSE}(+)$ groups, clinical improvements were accompanied by highly significant $(\mathrm{df}=1,126$; all $p<.0001$ ) changes from baseline to termination on the following rating scales: HRS-D from $25.3 \pm 4.4$ to $12.8 \pm$ $8.7, F=20.94$; HMES, $23.2 \pm 4.7$ to $11.8 \pm 8.1, F=19.75$; CGAE, $3.16 \pm 0.77$ to $4.10 \pm 0.91, F=8.13$; and CGAS, $4.3 \pm 0.50$ to $2.74 \pm 1.4, F=13.85$. In the $\operatorname{CSE}(-)$ patients $(\mathrm{df}=1,126)$, improvements on the clinical scales were relatively smaller and, except for CGAE, also statistically significant: HRS-D, $22.7 \pm 2.9$ to $17.3 \pm 8.8, F=$ $5.00, p<.0001$; HMES, $19.1 \pm 4.3$ to $14.7 \pm 7.9, F=4.61$, $p<.0001$; CGAS, $4.11 \pm 0.32$ to $3.37 \pm 1.30, F=3.11$, $p=.005$; and CGAE, $3.10 \pm 0.88$ to $3.21 \pm 1.23, F=0.38$, NS. There also were highly significant group-by-time interactions ( $\mathrm{df}=7,252$; all $p<.001$ ) between the two groups over time: HRS-D, $F=4.59$; HMES, $F=5.24$; CGAS, $F=3.68$; and CGAE, $F=4.13$.

\section{DISCUSSION}

The pharmacokinetics and pharmacodynamics of NT have been fairly well studied in the general population, and it has therefore been used as the drug of reference for newer antidepressants (Katz et al. 1989). Placebocontrolled studies with NT in the elderly are, however, small in number. In this multicenter study, NT was found to be significantly more effective than placebo in the treatment of elderly depressed patients, although it was associated with more side effects of the anticholinergic type than placebo. Several findings are noteworthy about some aspects of the pharmacokinetics and pharmacodynamics of NT in this patient population: relationship between dosage of NT and therapeutic window, timing of steady state, metabolism of NT to its 10hydroxy analog over the period of the study, efficacy of the drug treatment, and the relationship between serum levels of nortriptyline and the 10-hydroxy-metabolites and incidence/severity of peripheral anticholinergic side effects.

\section{Dosage and Therapeutic Window}

A major concern in the treatment of elderly depressed patients with tricyclic antidepressant is the occurrence of side effects, so that there may be a tendency to use suboptimal doses. In this context, the previously defined therapeutic window may be useful, although it has not been validated in the elderly. A therapeutic window with serum levels between 50 and $170 \mathrm{ng} / \mathrm{ml}$ has been proposed (Asberg et al. 1971; Kragh-Sorensen et al. 1973; Kragh-Sorensen and Larsen 1980). At week 1, serum NT levels in the majority of patients $(81 \%)$ were within the therapeutic range, with a $75-\mathrm{mg}$ daily dose.

Table 1. Anticholingeric Side Effects and Levels of Serum Nortriptyline and Serum OHNortriptyline at End of Weeks 1 and 3

\begin{tabular}{|c|c|c|c|c|c|c|c|c|c|c|c|c|c|}
\hline \multirow[b]{2}{*}{ Week } & \multirow[b]{2}{*}{ Side Effect } & \multicolumn{4}{|c|}{$\begin{array}{c}\text { Nortriptyline } \\
\text { (NT ng/ml) }\end{array}$} & \multicolumn{4}{|c|}{ OH-NT (ng/ml) } & \multicolumn{4}{|c|}{ OH-NT/NT } \\
\hline & & Mean & SD & $\mathbf{N}$ & $p^{*}$ & Mean & SD & $\mathbf{N}$ & $p^{*}$ & Mean & $\mathrm{SD}$ & $\mathbf{N}$ & $p^{*}$ \\
\hline 1 & $\operatorname{CSE}(+)$ & 124 & 74 & 16 & .05 & 118 & 49 & 9 & NS & 0.94 & 0.5 & 9 & .07 \\
\hline 1 & $\operatorname{CSE}(-)$ & 82 & 35 & 16 & & 100 & 55 & 10 & & 1.5 & 1.1 & 10 & \\
\hline 3 & $\operatorname{CSE}(+)$ & 133 & 64 & 15 & .05 & 127 & 51 & 11 & NS & 0.83 & 0.3 & 11 & .02 \\
\hline 3 & $\operatorname{CSE}(-)$ & 93 & 32 & 14 & & 140 & 78 & 11 & & 1.5 & 0.9 & 11 & \\
\hline
\end{tabular}

* ANOVA between $\operatorname{CSE}(+)$ (moderate or severe anticholinergic side effects) and $\operatorname{CSE}(-)$ (no or mild anticholinergic side effects). 
The rate of clinical remission in this study was relatively high compared to placebo. These results tend to lend support for a similar therapeutic window in the depressed elderly.

\section{Half-life and Metabolism}

The half-life of NT in the general population is in the range of 15 to 16 hours (Rubin et al. 1985). It can therefore be assumed that a lag time of 1 week would be amply sufficient to achieve $90 \%$ of serum NT levels at steady state. However, Dawling et al. (1980) calculated the half-life to be 45 hours in elderly patients (68-100 years old) on $75 \mathrm{mg}$ of oral NT. In our study, it appears that the interval required for serum levels of NT to achieve steady state occurs between 1 and 3 weeks, because there was a significant increase in the NT levels in this interval. In subsequent studies, measurements at more frequent intervals should give a more accurate assessment of the half-life of NT in the elderly. The longer half-life observed here can be attributed to delayed absorption and reduced gut motility rather than slowed metabolism, because conversion to the 10-hydroxy metabolite is not dissimilar from what it is in younger patients.

Previous studies on the rates of metabolic transformation of NT in elderly patients gave conflicting results. Some authors suggested that it was much slower than in younger patients (Bertilsson et al. 1979; Young et al. 1984, 1985), while others found no differences (Katz et al. 1989; Kanba et al. 1992); the mean ratios of OH-NT to NT levels reported ranged from 1.2 to 2.7 . Young et al. (1987) attributed the increase in serum 10-hydroxymetabollite levels observed in elderly patients to reduced renal clearance. In our study, such a relationship was not found; the average OH-NT to NT ratio observed was just under 1 , not unlike that reported for younger patients, and was independent of serum creatinine levels. A statistically significant and weak positive correlation was found between the parent drug and its 10-hydroxy metabolite at week 1 but not over the remainder of the study period. This is in agreement with the findings of Gram et al. (1989) who found no significant correlation between NT and the major form (i.e., E-isomer) of the 10-hydroxy metabolite, although they did find a highly significant positive correlation with the minor Z-isomer at steady state. In our study, only the major form was measured, as the minor $Z$-isomer was present in very small concentrations.

On the other hand, there were significant and strong positive associations found among levels of the 10-hydroxy-metabolites at weeks 1 and 3 and at termination. This association was also found for the parent drug, indicating little, if any, change in kinetics over the period of the study, maybe because this study was part of a clinical trail that followed strict medical admission criteria (Nair et al. 1995).

\section{Relationship between NT, OH-NT Levels, and Side Effects}

As mentioned, the 75-mg NT dose was effective but was also accompanied by anticholinergic side effects. These appeared to be related to the levels of serum NT. Thus, when patients with moderate to severe anticholinergic side effects $[\mathrm{CSE}(+)]$ were compared to those with mild or none $[\mathrm{CSE}(-)]$, the former had significantly higher serum NT levels (Table 1). On the other hand, they showed no significant differences in serum metabolite levels. However, the rate of metabolic transformation appears to be a contributing factor. Fast metabolizers, that is, those with high ratios of serum levels of metabolites to the parent drug, appeared to suffer less anticholinergic side effects. Thus, CSE $(-)$ patients had significantly higher ratios than $\operatorname{CSE}(+)$ patients. Furthermore, there was a significant positive correlation between levels of metabolites and tolerance (CGAT). The relationship found between NT and OH-NT and anticholinergic side effects confirm preclinical studies on these two compounds. NT is known to have a high affinity for muscarinic receptors. In comparison, its metabolite, OH-NT, has been found to have a 10 to 12 times lower affinity for muscarinic receptors in the heart, brain, parotid gland, urinary bladder, and ileum in the guinea pig (Nilvebrant and Nordin 1991). In a recent clinical trial on the efficacy of $\mathrm{OH}-\mathrm{NT}$ as an antidepressant, the compound was also found to have little anticholinergic side effects (Nordin et al. 1991).

High levels of the 10-hydroxy metabolites have been correlated with ECG abnormalities. Young et al. (1985) found that patients with a mean ratio of 2.7 for serum $\mathrm{OH}-\mathrm{NT} / \mathrm{NT}$ had changes in PR measurements compared to those with a mean ratio of 1.5. In our study, none of the patients had PR changes or any ECG abnormalities; the average ratio of serum OH-NT/NT was less than 1. McCue et al. (1989) reported no correlations between changes in PR and QRS and QTc measurements with either serum NT or OH-NT levels, in an elderly population given various doses of nortriptyline. The mean total serum levels of both the parent drug and its metabolite were, however, lower than those reported by Young et al. (1985).

\section{Relationship between Efficacy and Side Effects}

NT has been shown to be an effective antidepressant, but there is considerable variation in the incidence and severity of anticholinergic side effects. In the present study, we have found an association between efficacy and side effects. Thus, on several measures of clinical efficacy (HRS-D, HMES, CGAE, CGAS), CSE(+) patients showed a greater clinical improvement than CSE $(-)$ patients on these measures over the period of the study. However, the overall rate of dropouts due to intolerance of these side effects was small. 
In conclusion, $75 \mathrm{mg}$ of NT administered daily in a divided dose appears to be effective in the treatment of major depression in the elderly. Higher serum levels of NT and slow metabolic transformation were associated with more severe, but tolerable, anticholinergic side effects.

\section{ACKNOWLEDGMENTS}

The authors wish to thank the clinical monitors and laboratory personnel for their excellent help and Roche International for financial support.

\section{REFERENCES}

American Psychiatric Association (1987): Diagnostic and statistical manual of mental disorders, ed. 3, rev. Washington, DC, American Psychiatric Association

Asberg M, Cronholm B, Sjoqvist F, Tuck D (1971): Relationship between plasma level and therapeutic effect of nortriptyline. Br Med J 3:331-334

Bech P (1981): Rating scales for affective disorders: Their validity and consistency. Acta Psychiatr Scand 64 (suppl 295):1-101

Bertilsson L, Mellstrom B, Sjoqvist F (1979): Pronounced inhibition of noradrenaline uptake by 10-hydroxymetabolites of nortriptyline. Life Sci 25:1285-1292

Dawling S, Crome P, Braithwaite R (1980): Pharmacokinetics of single oral doses of nortriptyline in depressed elderly hospital patients and young healthy volunteers. Clin Pharmacokinetics 5:394-401

Georgotas A, McCue RE, Hapworth W, Friedman E, Kim OM, Welkowitz J, Chang I, Cooper TB (1986): Comparative efficacy and safety of MAOIs versus TCAs in treating depression in the elderly. Biol Psychiatry 21:11551166

Georgotas A, McCue RE, Cooper T, Chang I, Mir P, Welkowit7. R (1987a): Clinical predictions of response to antidepressants in elderly patients. Biol Psychiatry 22:733-740

Georgotas A, McCue RE, Friedman E, Cooper TB (1987b): Response of depressive symptoms to nortriptyline, phenelzine and placebo. Br J Psychiatry 151:102-106

Gram LF, Brosen K, Kragh-Sorensen P, Christensen P (1989): Steady-state plasma levels of E- and Z-10-OH-nortriptyline in nortriptyline-treated patients: Significance of concurrent medication and the sparteine oxidation type. Ther Drug Monit 11(5):508-514

Hamilton M (1960): A rating scale for depression. J Neurol Neurosurg Psychiatry 23:56-62

Kanba S, Matsumoto K, Nibuya M, Suzuki E, Kinoshita N, Shintani F, Yagi G (1992): Nortriptyline response in elderly depressed patients. Prog Neuro Psychopharmacol Biol Psychiatry 16:301-309
Katz IR, Simpson GM, Jethanandani V, Cooper T, Muhly C (1989): Steady state pharmacokinetics of nortriptyline in the frail elderly. Neuropsychopharmacology 2:229-236

Kiltgaard NA (1977): Determination of therapeutic concentrations of doxepin and desmethyldoxepin in human serum by high performance liquid chromatography. Arch Pharm Chem Sci Ed 5:61-66

Kragh-Sorensen P, Larsen N-E (1980): Factors influencing nortriptyline steady-state kinetics: Plasma and saliva levels. Clin Pharmacol Therap 28:796-803

Kragh-Sorensen P, Asberg M, Hansen E (1973): Plasma nortriptyline levels in endogenous depression. Lancet $1: 113-115$

McCue RE, Georgotas A, Nagachandran N, Basir MA, Go EA, Suckow RF, Cooper TB (1989): Plasma levels of nortriptyline and 10-hydroxynortriptyline and treatmentrelated electrocardiographic changes in the elderly depressed. J Psychiatr Res 23:73-79

Miller MD, Pollock BG, Rifai AH, Paradis CF, Perel JM, George C, Stack JA, Reynolds CF III (1991): Longitudinal analysis of nortriptyline side effects in elderly depressed patients. J Geriatr Psychiatry Neurol 4:226230

Nair NPV, Amin M, Holm P, Katona C, Klitgaard N, Ng Ying Kin NMK, Kragh-Sorensen P, Kuhn H, Leek CA, Stage KB (1995): Moclobemide and nortriptyline in elderly depressed patients. A randomized, multicentre trial against placebo. J Affect Dis 33(1):1-9

Nilvebrant L, Nordin C (1991): Affinity of nortriptyline and its E-10-hydroxy metabolite for muscarinic receptors. Pharmacol Toxicol 68(1):64-67

Nordin C, Bertilsson L, Dahl M-L, Resul B, Toresson G, Sjoqvist F (1991): Treatment of depression with E-10hydroxynortriptyline-A pilot study on biochemical effects and pharmacokinetics. Psychopharmacology $103: 287-290$

Potter WZS, Rudorfer MV, Manji H (1991): The pharmacologic treatment of depression. N Eng J Med 325:633-642

Rafaelson OJ, Clemmesen L, Lund H, Mikkelsen PL, Bolwig TG (1981): Comparison of peripheral anti-cholinergic effects of antidepressants: Dry mouth. Recent advances in the treatment of depression. Acta Psychiatr Scand 63:364-369

Rubin EH, Bigss JT, Preskorn SH (1985): Nortriptyline pharmacokinetics and plasma levels: Implications for clinical practice J Clin Psychiatry 46:418-424

Young RC, Alexopoulos GC, Shamoian CA, Manley MW, Dhar AK, Kuff H (1984): Plasma-10-hydroxy-nortriptyline in elderly depressed patients. Clin Pharmacol Ther 35:540-544

Young RC, Alexopoulos GC, Shamoian CA, Kitt H (1985): Plasma-10-hydroxy-nortriptyline and EKG changes in elderly depressed patients. Am J Psychiatry 142:866-868

Young RC, Alexopoulos GC, Dhar AK, Kuff H (1987): Plasma 10-hydroxy-nortriptyline renal function in elderly depressives. Biol Psychiatry 22:1283-1287 